\title{
EXISTENCE AND STABILITY OF ANTI PERIODIC SOLUTION FOR FCNNS WITH VARIABLE COEFFICIENTS IN THE LEAKAGE TERMS
}

\author{
HONGMEI BAO \\ Faculty of Mathematics and Physics \\ Huaiyin Institute of Technology \\ Huai'an, Jiangsu 223003, P.R. CHINA
}

\begin{abstract}
This paper is concerned with the problem of anti periodic solution for a class of fuzzy cellular neural networks (FCNNs) with variable coefficients in the leakage terms. Using contraction mapping and fixed point theorem and differential inequality, we obtain some sufficient conditions to guarantee the existence and exponential stability of the anti periodic solution for this model. These results complement previously known publications. Moreover a numerical example is given to show effectiveness of results obtained.
\end{abstract}

AMS Subject Classification: 34K20, 34K13, 92B20

Key Words: fuzzy cellular neural networks, anti periodic solution, global exponential stability, variable coefficients, leakage terms

Received: October 23, 2018; Revised: February 21, 2019;

Published (online): February 25, $2019 \quad$ doi: $10.12732 /$ dsa.v28i2.4

Dynamic Publishers, Inc., Acad. Publishers, Ltd. https://acadsol.eu/dsa

\section{INTRODUCTION}

In this paper, we consider the problem of anti-periodic solution for fuzzy cellular neural networks (FCNNs) with variable coefficients in the leakage terms, for $i=$ $1,2, \cdots, n$, 


$$
\begin{aligned}
x_{i}^{\prime}(t)= & -c_{i}(t) x_{i}(t)+\sum_{j=1}^{n} a_{i j}(t) f_{j}\left(x_{j}\left(t-\tau_{i j}(t)\right)\right) \\
& +\bigwedge_{j=1}^{n} \alpha_{i j}(t) \int_{0}^{\infty} K_{i j}(s) g_{j}\left(x_{j}(t-s)\right) d s \\
& +\bigvee_{j=1}^{n} \beta_{i j}(t) \int_{0}^{\infty} K_{i j}(s) g_{j}\left(x_{j}(t-s)\right) d s+I_{i}(t),
\end{aligned}
$$

which has been successfully applied in many fields such as image and signal processing, pattern recognition, optimization and automatic control, see for example [1, 2]. Here the first term in each of the right sides of (1) is variously known as forgetting or leakage term (see for instance $[3,4]$ ), $n$ is the number of units in a neural networks, $x_{i}(t)$ corresponds to the state of the $i$-th unit at time $t . c_{i}(t)$, the leakage coefficient function, denotes the rate with which the $i$-th unit will reset its potential to the resting state in isolation when disconnected from the network and external input. $a_{i j}(t)$ represents the strength of the $j$-th unit on the $i$-th unit at time $t . \wedge$ and $\bigvee$ denote fuzzy AND and fuzzy OR operations, respectively. $\alpha_{i j}(t)$ and $\beta_{i j}(t)$ are the elements of fuzzy feedback MIN and fuzzy feedback MAX template at time $t$ respectively. $K_{i j}(s)$ and $\tau_{i j}(t) \geq 0$ are respectively transmission delay kernel and transmission delay. $f_{j}(\cdot)$ and $g_{j}(\cdot)$ are signal transmission functions. $I_{i}(t)$ are external input to the $i$-th unit at time $t . i, j=1,2, \cdots, n$.

As is all known, FCNNs first proposed by Yang and Yang [1, 2] is a new cellular neural networks model, which combined fuzzy AND and fuzzy OR operations with cellular neural networks. It is worth noting that FCNNs are different from T-S fuzzy neural networks which are based on a set of fuzzy rules to describe nonlinear system. Recently many studies have shown that FCNNs are useful in image processing and pattern recognition, and some interesting results have been published on stability of equilibrium and periodic solution of FCNNs with delays [5, 6, 7, 8, 9, 10, 11].

On the other hand, the existence and stability of anti-periodic solutions of dynamical system are also an very important topic characterizing the dynamical behavior of nonlinear differential equation. For example, the signal transmission process of neural networks can often be described as an anti-periodic process. The study on anti periodic solution of neural networks has theoretical value and also tremendous potential in applications (see $[12,13,14,15,16,17,18,19,20,21,22,23]$ and references therein). Furthermore, assuming that

$$
\inf _{t \in R} c_{i}(t)>0, \quad i=1,2, \cdots, n .
$$

In the past few decades, people have paid much attention to the stability on biological systems with variable coefficients appear in linearizations of population dynamics models with seasonal fluctuations, where during some seasons the death or harvesting 
rates may be greater than the birth rate (see $[24,25])$. Some interesting results on anti periodic solution of CNNs have been published in recent years. However, to the best of the authors knowledge, few authors have handled the anti-periodic solutions of FCNNs with variable coefficients in leakage terms. Motivated by these discussion above, we establish some sufficient conditions to guarantee the existence and exponential stability of anti-periodic solutions for (1) without (2). Particularly, our results not only generalize the existing ones in $[11,12,13,14,15,16,17,18]$, but also improve them. In fact, one can see the following Remarks 3.1 for details.

The rest of this paper is organized as follows. In Section 2, some preliminaries and basic assumptions are established. In Section 3, some sufficient conditions are derived for guaranteeing the existence and exponential stability of an anti-periodic solution for system (1). In Section 4, an numerical example is given to illustrate our results. Finally, a general conclusion is drawn in Section 5.

\section{PRELIMINARIES AND SOME ASSUMPTIONS}

In this section, we shall first recall some basic definitions and lemmas which are used in what follows.

Let $R^{n}$ be the set of all $n$ dimension real vectors. $x=\left(x_{1}, x_{2}, \cdots, x_{n}\right)^{T} \in R^{n}$ denotes a column vector (symbol ${ }^{T}$ denotes the transpose of a vector).

Let $x(t): R \rightarrow R$ be continuous at $t . x(t)$ is said to be $\omega$-anti periodic on $R$ if $x(t+\omega)=-x(t)$, for all $t \in R$. where $\omega$ is a positive constant. We let $|x|_{\infty}=$ $\max _{t \in[0, \omega]}|x(t)| . C(R, R)=\{x \mid x(t): R \rightarrow R$ be continuous in $t\}$ and $C^{\omega}:=\{x=$ $\left.\left\{x_{i}\right\}, x_{i} \in C(R, R), x_{i}(t+\omega)=-x_{i}(t)\right\}$ which is a Banach space endowed with the norm $\|\cdot\|$ defined by $\|x\|=\max _{1 \leq i \leq n}\left|x_{i}\right|_{\infty}$. Given a bounded and continuous function $g$ defined on $R, g^{+}=\sup _{t \in R} g(t), g^{-}=\inf _{t \in R} g(t)$.

A matrix or vector $A \geq 0$ means that all entries of $A$ are greater than or equal to zero. $A>0$ can be defined similarly. For matrices or vectors $A$ and $B, A \geq B$ (resp. $A>B$ ) means that $A-B \geq 0$ (resp. $A-B>0$ ).

The initial conditions associated with system (1) are of the form

$$
x_{i}(s)=\varphi_{i}(s), \quad s \in(-\infty, 0], i=1,2, \cdots, n .
$$

where $\varphi_{i}(\cdot)$ is a real-valued bounded and continuous function defined on $(-\infty, 0]$.

Throughout this paper, the following conditions hold 
(A1) For $i, j=1,2, \cdots, n, k=1,2, \cdots, v \in R$, there exists $\omega>0$ such that

$$
\left\{\begin{array}{l}
c_{i}(t+\omega)=c_{i}(t), \quad a_{i j}(t+\omega) f_{j}(-v)=-a_{i j}(t) f_{j}(v), \\
I_{i}(t+\omega)=-I_{i}(t), \alpha_{i j}(t+\omega)=-\alpha_{i j}(t), g_{j}(-v)=g_{j}(v), \\
\tau_{i j}(t+\omega)=\tau_{i j}(t), \beta_{i j}(t+\omega)=-\beta_{i j}(t),
\end{array}\right.
$$

(A2) For $j=1,2, \cdots, n$, the activation functions $f_{j}, g_{j}: R \rightarrow R$ are Lipchtiz continuous, i.e., there exist nonnegative constants $\mu_{j}, \nu_{j}$ such that, for $u, v \in R$,

$$
\left\{\begin{array}{l}
\left|f_{j}(u)-f_{j}(v)\right| \leq \mu_{j}|u-v|, \\
\left|g_{j}(u)-g_{j}(v)\right| \leq \nu_{j}|u-v| .
\end{array}\right.
$$

(A3) For $i=1,2, \cdots, n$ there exist a bounded continuous function $c^{*}: R \rightarrow(0,+\infty)$ and positive constants $G_{i}^{s}$ such that

$$
e^{-\int_{s}^{t} c_{i}(s) d s} \leq G_{i} e^{-\int_{s}^{t} c_{i}^{*}(s) d s}, t, s \in R, t>s .
$$

(A4) For $i, j=1,2, \cdots, n$, the delay kernel $K_{i j}:[0,+\infty) \rightarrow R$ are continuous and $\left|K_{i j}(t)\right| e^{\gamma t}$ are integrable on $R$, for a positive constant $\gamma$.

(A5) For $i=1,2, \cdots, n$, there exist constants $\delta_{i}$ and $\xi_{j}$ such that for $t>0$,

$$
\begin{aligned}
& \sup _{t \in R}\left\{-c_{i}^{*}(t)+G_{i}^{s}\left[\frac { 1 } { \xi _ { i } } \sum _ { j = 1 } ^ { n } \left(\left|a_{i j}(t)\right| \mu_{j}+\left(\left|\alpha_{i j}(t)\right|+\left|\beta_{i j}(t)\right|\right)\right.\right.\right. \\
& \left.\left.\left.\times \nu_{j} \int_{0}^{\infty}\left|K_{i j}(s)\right| d s\right) \xi_{j}\right]\right\}<-\delta_{i}<0 .
\end{aligned}
$$

Definition 1. The anti periodic solution $x^{*}(t)=\left(x_{1}^{*}(t), x_{2}^{*}(t), \cdots, x_{n}^{*}(t)\right)^{T}$ of system (1) with the initial value $\varphi^{*}=\left(\varphi_{1}^{*}, \varphi_{2}^{*}, \cdots, \varphi_{n}^{*}\right)^{T} \in C\left(R, R^{n}\right)$ is said to be globally exponentially stable, if there exist constants $\lambda>0$ and $M \geq 1$ such that

$$
\left|x_{i}(t)-x_{i}^{*}(t)\right| \leq M\left\|\varphi-\varphi^{*}\right\| e^{-\lambda t}, \forall t>0, i=1,2, \cdots, n .
$$

for every solution $x(t)=\left(x_{1}(t), x_{2}(t), \cdots, x_{n}(t)\right)^{T}$ of system (1.1) with initial value $\varphi \in C\left(R, R^{n}\right)$. where

$$
\left\|\varphi-\varphi^{*}\right\|=\sup _{-\infty<s \leq 0} \max _{1 \leq i \leq n}\left|\varphi_{i}(s)-\varphi_{i}^{*}(s)\right| .
$$

Lemma 1. [1] If $u$ and $v$ are two states of system (1.1), then we have

$$
\left|\bigwedge_{j=1}^{n} \alpha_{i j}(t) g_{j}(u)-\bigwedge_{j=1}^{n} \alpha_{i j}(t) g_{j}(v)\right| \leq \sum_{j=1}^{n}\left|\alpha_{i j}(t)\right|\left|g_{j}(u)-g_{j}(v)\right|
$$


and

$$
\left|\bigvee_{j=1}^{n} \beta_{i j}(t) g_{j}(u)-\bigvee_{j=1}^{n} \beta_{i j}(t) g_{j}(v)\right| \leq \sum_{j=1}^{n}\left|\beta_{i j}(t)\right|\left|g_{j}(u)-g_{j}(v)\right| .
$$

\section{MAIN RESULTS}

In this section, we discuss the existence and exponential stability for an anti-periodic solution of system (1.1).

Theorem 2. If assumptions (A1)-(A5) hold. Then there exists a unique $\omega$ anti periodic solution of system (1).

Proof. Set $y_{i}(t)=\xi_{i}^{-1} x_{i}(t)$. Then it follows from (1) that

$$
\begin{aligned}
y_{i}^{\prime}(t)= & -c_{i}(t) y_{i}(t)+\frac{1}{\xi_{i}} \sum_{j=1}^{n} a_{i j}(t)\left[f_{j}\left(\xi_{j} y_{j}\left(t-\tau_{i j}(t)\right)\right)\right. \\
& +\frac{1}{\xi_{i}} \bigwedge_{j=1}^{n} \alpha_{i j}(t) \int_{0}^{\infty} K_{i j}(s) g_{j}\left(\xi_{j} y_{j}(t-s)\right) d s+\frac{1}{\xi_{i}} I_{i}(t) \\
& +\frac{1}{\xi_{i}} \bigvee_{j=1}^{n} \beta_{i j}(t) \int_{0}^{\infty} K_{i j}(s) g_{j}\left(\xi_{j} y_{j}(t-s)\right) d s
\end{aligned}
$$

Thus, to prove Theorem 2, it suffices to show that system (6) has a unique $\omega$-antiperiodic solution.

Defining a continuous function $(\Gamma \varphi)_{i}(t), \varphi \in c^{\omega}$ as follows

$$
\begin{aligned}
(\Gamma \varphi)_{i}(t)= & \int_{-\infty}^{t} e^{-\int_{s}^{t} c_{i}(u) d u}\left[\frac{1}{\xi_{i}} \sum_{j=1}^{n} a_{i j}(s) f_{j}\left(\xi_{j} y_{j}\left(s-\tau_{i j}(s)\right)\right)\right. \\
& +\frac{1}{\xi_{i}} \bigwedge_{j=1}^{n} \alpha_{i j}(s) \int_{0}^{\infty} K_{i j}(v) g_{j}\left(\xi_{j} y_{j}(s-v)\right) d v+\frac{1}{\xi_{i}} I_{i}(s) \\
& \left.+\frac{1}{\xi_{i}} \bigvee_{j=1}^{n} \beta_{i j}(s) \int_{0}^{\infty} K_{i j}(v) g_{j}\left(\xi_{j} y_{j}(s-v)\right) d v\right] d s
\end{aligned}
$$

Next we prove that $\Gamma_{i}: C^{\omega} \rightarrow C^{\omega}$ is a contraction mapping. In fact, in view of (7), (A2),(A3) and (A5), using Lemma 1, for $\varphi, \phi \in C^{\omega}$, we have

$$
\begin{aligned}
& \left|(\Gamma \varphi)_{i}(t)-(\Gamma \phi)_{i}(t)\right| \\
= & \mid \int_{-\infty}^{t} e^{-\int_{s}^{t} c_{i}(u) d u}\left[\frac { 1 } { \xi _ { i } } \sum _ { j = 1 } ^ { n } a _ { i j } ( s ) \left(f_{j}\left(\xi_{j} \varphi_{j}\left(s-\tau_{i j}(s)\right)\right)\right.\right.
\end{aligned}
$$




$$
\begin{aligned}
& \left.-f_{j}\left(\xi_{j} \phi_{j}\left(s-\tau_{i j}(s)\right)\right)\right) \\
& +\frac{1}{\xi_{i}}\left(\bigwedge_{j=1}^{n} \alpha_{i j}(s) \int_{0}^{\infty} K_{i j}(v) g_{j}\left(\xi_{j} \varphi_{j}(s-v)\right) d v\right. \\
& \left.-\bigwedge_{j=1}^{n} \alpha_{i j}(s) \int_{0}^{\infty} K_{i j}(v) g_{j}\left(\xi_{j} \phi_{j}(s-v)\right) d v\right) \\
& +\frac{1}{\xi_{i}}\left(\bigvee_{j=1}^{n} \beta_{i j}(s) \int_{0}^{\infty} K_{i j}(v) g_{j}\left(\xi_{j} \varphi_{j}(s-v)\right) d v\right. \\
& \left.\left.-\bigvee_{j=1}^{n} \beta_{i j}(s) \int_{0}^{\infty} K_{i j}(v) g_{j}\left(\xi_{j} \phi_{j}(s-v)\right) d v\right)\right] d s \\
& \leq \int_{-\infty}^{t} e^{-\int_{s}^{t} c_{i}(u) d u}\left[\frac{1}{\xi_{i}} \sum_{j=1}^{n}\left|a_{i j}(s)\right| \mu_{j} \xi_{j}\left|\varphi_{j}\left(s-\tau_{i j}(s)\right)-\phi_{j}\left(s-\tau_{i j}(s)\right)\right|\right. \\
& +\frac{1}{\xi_{i}} \sum_{j=1}^{n}\left(\left|\alpha_{i j}(s)\right|+\left|\beta_{i j}(s)\right|\right) \\
& \left.\times \int_{0}^{\infty}\left|K_{i j}(v)\right| \nu_{j} \xi_{j}\left|\varphi_{j}(s-v)-\phi_{j}(s-v)\right| d v\right] d s \\
& \leq \int_{-\infty}^{t} e^{-\int_{s}^{t} c_{i}^{*}(u) d u} G_{i}^{S}\left[\frac { 1 } { \xi _ { i } } \sum _ { j = 1 } ^ { n } \left(\left|a_{i j}(s)\right| \mu_{j}+\left(\left|\alpha_{i j}(s)\right|+\left|\beta_{i j}(s)\right|\right) \nu_{j}\right.\right. \\
& \left.\left.\times \int_{0}^{\infty}\left|K_{i j}(v)\right| d v\right) \xi_{j}\right] d s\|\varphi-\phi\| \\
& \leq \int_{-\infty}^{t} e^{-\int_{s}^{t} c_{i}^{*}(u) d u}\left(c_{i}^{*}(s)-\delta_{i}\right) d s\|\varphi-\phi\| \\
& \leq \int_{-\infty}^{t} e^{-\int_{s}^{t} c_{i}^{*}(u) d u}\left(c_{i}^{*}(s)-\frac{\delta_{i}}{3}\right) d s\|\varphi-\phi\| \\
& \leq\left[\int_{-\infty}^{t} e^{-\int_{s}^{t} c_{i}^{*}(u) d u} d\left(-\int_{s}^{t} c_{i}^{*}(u) d u\right)-\frac{\delta_{i}}{3} \int_{-\infty}^{t} e^{-\int_{s}^{t} c_{i}^{*}(u) d u} d s\right]\|\varphi-\phi\| \\
& \leq\left(1-\frac{\delta_{i}}{3 c_{i}^{*+}}\right)\|\varphi-\phi\|
\end{aligned}
$$

From (A5), we have

$$
0<\max _{1 \leq i \leq n}\left(1-\frac{\delta_{i}}{3 c_{i}^{*+}}\right)<1 .
$$

which, together with (7), yields

$$
\|\Gamma \varphi-\Gamma \phi\| \leq \max _{1 \leq i \leq n}\left(1-\frac{\delta_{i}}{3 c_{i}^{*+}}\right)\|\varphi-\phi\| .
$$


Namely, $\Gamma$ is a contraction mapping. Therefore, the mapping $\Gamma$ possesses a unique fixed point $y^{*} \in C^{\omega}$ and $\Gamma y^{*}=y^{*}$. Thus

$$
\begin{aligned}
y_{j}^{*}(t)= & \int_{-\infty}^{t} e^{-\int_{s}^{t} c_{i}(u) d u}\left[\frac{1}{\xi_{i}} \sum_{j=1}^{n} a_{i j}(s) f_{j}\left(\xi_{j} y_{j}^{*}\left(s-\tau_{i j}(s)\right)\right)\right. \\
& +\frac{1}{\xi_{i}} \bigwedge_{j=1}^{n} \alpha_{i j}(s) \int_{0}^{\infty} K_{i j}(v) g_{j}\left(\xi_{j} y_{j}^{*}(s-v)\right) d v \\
& \left.+\frac{1}{\xi_{i}} \bigvee_{j=1}^{n} \beta_{i j}(s) \int_{0}^{\infty} K_{i j}(v) g_{j}\left(\xi_{j} y_{j}^{*}(s-v)\right) d v+\frac{1}{\xi_{i}} I_{i}(s)\right] d s
\end{aligned}
$$

which is equivalent to

$$
\begin{aligned}
y_{i}^{*^{\prime}}(t)= & -c_{i}(t) y_{i}^{*}(t)+\frac{1}{\xi_{i}} \sum_{j=1}^{n} a_{i j}(t)\left[f_{j}\left(\xi_{j} y_{j}^{*}\left(t-\tau_{i j}(t)\right)\right)\right. \\
& +\frac{1}{\xi_{i}} \bigwedge_{j=1}^{n} \alpha_{i j}(t) \int_{0}^{\infty} K_{i j}(s) g_{j}\left(\xi_{j} y_{j}^{*}(t-s)\right) d s \\
& +\frac{1}{\xi_{i}} \bigvee_{j=1}^{n} \beta_{i j}(t) \int_{0}^{\infty} K_{i j}(s) g_{j}\left(\xi_{j} y_{j}^{*}(t-s)\right) d s+\frac{1}{\xi_{i}} I_{i}(t)
\end{aligned}
$$

By the equivalence of (1) and (6), one can show that system (1) has a unique $\omega$-antiperiodic solution $x^{*}(t)=\xi_{i} y^{*}(t)$. This completes the proof of Theorem 2 .

Theorem 3. Suppose that all conditions in Theorem 2 are satisfied. Then system (1) has exactly one $\omega$-anti-periodic solution $x^{*}(t)$ which is globally exponentially stable.

Proof. By Theorem 2, system (1) has a unique $\omega$-anti-periodic solution $x^{*}(t)$. Let $x(t)=\left(x_{1}(t), x_{2}(t), \cdots, x_{n}(t)\right)^{T}$ be a solution of (1.1) associated with initial value $\varphi(t)=\left(\varphi_{1}(t), \varphi_{2}(t), \cdots, \varphi_{n}(t)\right)^{T}$ satisfying $(3)$. Let $u_{i}(t)=\frac{1}{\xi_{i}}\left(x_{i}(t)-x_{i}^{*}(t)\right)$. Then

$$
\begin{aligned}
u_{i}^{\prime}(t)= & -c_{i}(t) u_{i}(t)+\frac{1}{\xi_{i}} \sum_{j=1}^{n} a_{i j}(t)\left[f_{j}\left(x_{j}\left(t-\tau_{i j}(t)\right)\right)-f_{j}\left(x_{j}\left(t-\tau_{i j}(t)\right)\right)\right] \\
& +\frac{1}{\xi_{i}}\left[\bigwedge_{j=1}^{n} \alpha_{i j}(t) \int_{0}^{\infty} K_{i j}(s) g_{j}\left(x_{j}(t-s)\right) d s\right. \\
& \left.-\bigwedge_{j=1}^{n} \alpha_{i j}(t) \int_{0}^{\infty} K_{i j}(s) g_{j}\left(x_{j}^{*}(t-s)\right) d s\right] \\
& +\frac{1}{\xi_{i}}\left[\bigvee_{j=1}^{n} \beta_{i j}(t) \int_{0}^{\infty} K_{i j}(s) g_{j}\left(y_{j}(t-s)\right) d s\right.
\end{aligned}
$$




$$
\left.-\bigvee_{j=1}^{n} \beta_{i j}(t) \int_{0}^{\infty} K_{i j}(s) g_{j}\left(y_{j}(t-s)\right) d s\right]
$$

Define a continuous function $\Psi_{i}(r)$ by setting,for $r \in[0, \gamma]$,

$$
\begin{aligned}
\Psi_{i}(r)= & r-c_{i}^{*}(t)+G_{i}\left[\frac { 1 } { \xi _ { i } } \sum _ { j = 1 } ^ { n } \left(\left|a_{i j}(t)\right| \mu_{j} e^{r \tau_{i j}(t)}+\left(\left|\alpha_{i j}(t)\right|+\left|\beta_{i j}(t)\right|\right) \nu_{j}\right.\right. \\
& \left.\left.\times \int_{0}^{\infty}\left|K_{i j}(v)\right| e^{r v} d v\right) \xi_{j}\right]
\end{aligned}
$$

According to (A4), we have

$$
\begin{aligned}
\Psi_{i}(0)= & -c_{i}^{*}(t)+G_{i}\left[\frac { 1 } { \xi _ { i } } \sum _ { j = 1 } ^ { n } \left(\left|a_{i j}(t)\right| \mu_{j}+\left(\left|\alpha_{i j}(t)\right|+\left|\beta_{i j}(t)\right|\right) \nu_{j}\right.\right. \\
& \left.\left.\times \int_{0}^{\infty}\left|K_{i j}(v)\right| d v\right) \xi_{j}\right] \leq-\delta_{i}<0
\end{aligned}
$$

Since $\Psi_{i}(r)$ is continuous, we can choose a constant $\lambda \in\left(0, \min \left\{\gamma, \min _{1 \leq i \leq n}\left(c_{i}^{*-}\right)\right\}\right)$ such that

$$
\begin{aligned}
\Psi_{i}(\lambda)= & \lambda-c_{i}^{*}(t)+G_{i}\left[\frac { 1 } { \xi _ { i } } \sum _ { j = 1 } ^ { n } \left(\left|a_{i j}(t)\right| \mu_{j} e^{\lambda \tau_{i j}(t)}+\left(\left|\alpha_{i j}(t)\right|+\left|\beta_{i j}(t)\right|\right) \nu_{j}\right.\right. \\
& \left.\left.\times \int_{0}^{\infty}\left|K_{i j}(v)\right| e^{\lambda v} d v\right) \xi_{j}\right]<0
\end{aligned}
$$

Let $\left\|\varphi-x^{*}\right\|=\sup _{t \leq 0} \max _{1 \leq i \leq n}\left\{\frac{1}{\xi_{i}}\left|\varphi_{i}(t)-x_{i}^{*}(t)\right|\right\}$ and $M=\sum_{i=1}^{n} G_{i}+1$. Consequently, for any $\varepsilon>0$ it is clear that, for all $t \in(-\infty, 0]$,

$$
\|u(t)\|<\left(\left\|\varphi-x^{*}\right\|+\varepsilon\right) e^{-\lambda t}<M\left(\left\|\varphi-x^{*}\right\|+\varepsilon\right) e^{-\lambda t}
$$

In the following, we will show, for $t \geq 0,(12)$ hold true. Otherwise, there must exist $i \in\{1,2, \cdots, n\}$ and $\theta>0$ such that, for $t \in(-\infty, \theta)$,

$$
\left|u_{i}(\theta)\right|=M\left(\left\|\varphi-x^{*}\right\|+\varepsilon\right) e^{\lambda \theta}, \quad\|u(t)\|<M\left(\left\|\varphi-x^{*}\right\|+\varepsilon\right) e^{\lambda t} .
$$

Noting that (9), we have, for $s \in[0, t], t \in[0, \theta]$,

$$
\begin{aligned}
u_{i}^{\prime}(s) & +c_{i}(s) u_{i}(s)=\frac{1}{\xi_{i}} \sum_{j=1}^{n} a_{i j}(s)\left[f_{j}\left(x_{j}\left(s-\tau_{i j}(s)\right)\right)-f_{j}\left(x_{j}\left(s-\tau_{i j}(s)\right)\right)\right] \\
& +\frac{1}{\xi_{i}}\left[\bigwedge_{j=1}^{n} \alpha_{i j}(s) \int_{0}^{\infty} K_{i j}(v) g_{j}\left(x_{j}(s-v)\right) d v\right.
\end{aligned}
$$




$$
\begin{array}{r}
\left.-\bigwedge_{j=1}^{n} \alpha_{i j}(s) \int_{0}^{\infty} K_{i j}(v) g_{j}\left(x_{j}^{*}(s-v)\right) d v\right] \\
+\frac{1}{\xi_{i}}\left[\bigvee_{j=1}^{n} \beta_{i j}(s) \int_{0}^{\infty} K_{i j}(v) g_{j}\left(y_{j}(s-v)\right) d s\right. \\
\left.-\bigvee_{j=1}^{n} \beta_{i j}(s) \int_{0}^{\infty} K_{i j}(v) g_{j}\left(y_{j}(s-v)\right) d v\right]
\end{array}
$$

Multiplying both sides of (14) by $e^{\int_{0}^{s} c_{i}(u) d u}$, and integrating it on $[0, t]$, we have

$$
\begin{gathered}
u_{i}(t)=u_{i}(0) e^{-\int_{0}^{t} c_{i}(u) d u} \\
+\int_{0}^{t} e^{-\int_{s}^{t} c_{i}(u) d u}\left\{\frac{1}{\xi_{i}} \sum_{j=1}^{n} a_{i j}(s)\left[f_{j}\left(x_{j}\left(s-\tau_{i j}(s)\right)\right)-f_{j}\left(x_{j}\left(s-\tau_{i j}(s)\right)\right)\right]\right. \\
+\frac{1}{\xi_{i}}\left[\bigwedge_{j=1}^{n} \alpha_{i j}(s) \int_{0}^{\infty} K_{i j}(v) g_{j}\left(x_{j}(s-v)\right) d v\right. \\
\left.-\bigwedge_{j=1}^{n} \alpha_{i j}(s) \int_{0}^{\infty} K_{i j}(v) g_{j}\left(x_{j}^{*}(s-v)\right) d v\right] \\
\quad \frac{1}{\xi_{i}} \bigvee_{j=1}^{n} \beta_{i j}(s) \int_{0}^{\infty} K_{i j}(v) g_{j}\left(y_{j}(s-v)\right) d s \\
\left.-\bigvee_{j=1}^{n} \beta_{i j}(s) \int_{0}^{\infty} K_{i j}(v) g_{j}\left(y_{j}(s-v)\right) d v\right]
\end{gathered}
$$

for $t \in[0, \theta]$. Thus we have

$$
\begin{aligned}
& \left|u_{i}(\theta)\right|=\mid u_{i}(0) e^{-\int_{0}^{\theta} c_{i}(u) d u} \\
& +\int_{0}^{\theta} e^{-\int_{s}^{\theta} c_{i}(u) d u}\left\{\frac{1}{\xi_{i}} \sum_{j=1}^{n} a_{i j}(s)\left[f_{j}\left(x_{j}\left(s-\tau_{i j}(s)\right)\right)-f_{j}\left(x_{j}\left(s-\tau_{i j}(s)\right)\right)\right]\right. \\
& +\frac{1}{\xi_{i}}\left[\bigwedge_{j=1}^{n} \alpha_{i j}(s) \int_{0}^{\infty} K_{i j}(v) g_{j}\left(x_{j}(s-v)\right) d v\right. \\
& \left.-\bigwedge_{j=1}^{n} \alpha_{i j}(s) \int_{0}^{\infty} K_{i j}(v) g_{j}\left(x_{j}^{*}(s-v)\right) d v\right] \\
& +\frac{1}{\xi_{i}}\left[\bigvee_{j=1}^{n} \beta_{i j}(s) \int_{0}^{\infty} K_{i j}(v) g_{j}\left(y_{j}(s-v)\right) d s\right.
\end{aligned}
$$




$$
\begin{aligned}
& \left.\left.-\bigvee_{j=1}^{n} \beta_{i j}(s) \int_{0}^{\infty} K_{i j}(v) g_{j}\left(y_{j}(s-v)\right) d v\right]\right\} d s \\
& \leq\left(\left\|\varphi-x^{*}\right\|+\varepsilon\right) G_{i} e^{-\int_{0}^{\theta} c_{i}^{*}(u) d u} \\
& +\int_{0}^{\theta} e^{-\int_{s}^{\theta} c_{i}(u) d u} G_{i}\left\{\frac{1}{\xi_{i}} \sum_{j=1}^{n} a_{i j}(s) \mu_{j} \xi_{j}\left|u_{j}\left(s-\tau_{i j}(s)\right)\right|\right. \\
& \left.+\frac{1}{\xi_{i}} \sum_{j=1}^{n}\left(\left|\alpha_{i j}(s)\right|+\left|\beta_{i j}(s)\right|\right) \int_{0}^{\infty}\left|K_{i j}(v)\right| \nu_{j} \xi_{j}\left|u_{j}(s-v)\right| d v\right\} d s \\
& \leq\left(\left\|\varphi-x^{*}\right\|+\varepsilon\right) G_{i} e^{-\int_{0}^{\theta} c_{i}^{*}(u) d u} \\
& +\int_{0}^{\theta} e^{-\int_{s}^{\theta} c_{i}^{*}(u) d u} G_{i}\left\{\frac{1}{\xi_{i}} \sum_{j=1}^{n} a_{i j}(s) \mu_{j} \xi_{j}\left(\left\|\varphi-x^{*}\right\|+\varepsilon\right) e^{-\lambda\left(s-\tau_{i j}(s)\right)}\right. \\
& +\frac{1}{\xi_{i}} \sum_{j=1}^{n}\left(\left|\alpha_{i j}(s)\right|+\left|\beta_{i j}(s)\right|\right) \\
& \left.\times \int_{0}^{\infty}\left|K_{i j}(v)\right| \nu_{j} \xi_{j} M\left(\left\|\varphi-x^{*}\right\|+\varepsilon\right) e^{-\lambda(s-v)} d v\right\} d s \\
& \leq\left(\left\|\varphi-x^{*}\right\|+\varepsilon\right) e^{-\lambda \theta} G_{i} e^{-\int_{0}^{\theta}\left(c_{i}^{*}(u)-\lambda\right) d u} \\
& +\int_{0}^{\theta} e^{-\int_{s}^{\theta}\left(c_{i}^{*}(u)-\lambda\right) d u} G_{i}\left\{\frac{1}{\xi_{i}} \sum_{j=1}^{n} a_{i j}(s) \mu_{j} \xi_{j}\left(\left\|\varphi-x^{*}\right\|+\varepsilon\right) e^{-\lambda \tau_{i j}(s)}\right. \\
& \left.+\frac{1}{\xi_{i}} \sum_{j=1}^{n}\left(\left|\alpha_{i j}(s)\right|+\left|\beta_{i j}(s)\right|\right) \int_{0}^{\infty}\left|K_{i j}(v)\right| \nu_{j} \xi_{j} e^{\lambda v} d v\right\} d s \\
& \times M\left(\left\|\varphi-x^{*}\right\|+\varepsilon\right) e^{-\lambda \theta} \\
& \leq\left(\left\|\varphi-x^{*}\right\|+\varepsilon\right) e^{-\lambda \theta} G_{i} e^{-\int_{0}^{\theta}\left(c_{i}^{*}(u)-\lambda\right) d u} \\
& +\int_{0}^{\theta} e^{-\int_{s}^{\theta}\left(c_{i}^{*}(u)-\lambda\right) d u}\left(c_{i}^{*}(s)-\lambda\right) d s M\left(\left\|\varphi-x^{*}\right\|+\varepsilon\right) e^{-\lambda \theta} \\
& =M\left(\left\|\varphi-x^{*}\right\|+\varepsilon\right) e^{-\lambda \theta}\left[1-\left(1-\frac{G_{i}}{M}\right) e^{-\int_{0}^{\theta}\left(c_{i}^{*}(u)-\lambda\right) d u}\right] \\
& <M\left(\left\|\varphi-x^{*}\right\|+\varepsilon\right) e^{-\lambda \theta}
\end{aligned}
$$

This contradicts the first equation of (13). Let $\varepsilon \rightarrow 0^{+}$, we have $\|u(t)\| \leq M \| \varphi-$ $x^{*} \| e^{-\lambda t}$ for all $t>0$. The proof of Theorem 3 is completed.

Remark 3.1 In compared with [14], the activation function $f_{j}(\cdot), g_{j}(\cdot)$ only satisfy Lipschtiz continuous and need not to be bounded and differentiable. The results obtained dependents on system parameters. Moreover the system (1) contains fuzzy AND and fuzzy OR template. One can observe that all the results in $[12,13,15,16$, 
$17,18,19,20,21,22]$ and the references therein can not be applicable to system (1). Therefore, the results obtained are new and generalize the results of publication.

\section{NUMERICAL EXAMPLE}

In this section, we give a numerical example to illustrate effectiveness of the results obtained.

Example Considering the following two neurons fuzzy cellular neural networks with variable coefficients in the leakage terms.

$$
\begin{aligned}
x_{i}^{\prime}(t)= & -c_{i}(t) x_{i}(t)+\sum_{j=1}^{2} a_{i j}(t) f_{j}\left(x_{j}\left(t-\tau_{i j}(t)\right)\right) \\
& +\bigwedge_{j=1}^{2} \alpha_{i j}(t) \int_{0}^{\infty} K_{i j}(u) g_{j}\left(x_{j}(t-u)\right) d u \\
& +\bigvee_{j=1}^{2} \beta_{i j}(t) \int_{0}^{\infty} K_{i j}(u) g_{j}\left(x_{j}(t-u)\right) d u+I_{i}(t),
\end{aligned}
$$

where $i=1,2$,

$$
\begin{gathered}
c_{1}(t)=3+\sin 20 t, c_{2}(t)=3+\cos 20 t, c_{1}^{*}(t)=c_{2}^{*}(t)=3, G_{1}=G_{2}=e^{\frac{1}{10}} . \\
a_{11}(t)=a_{21}(t)=\frac{1}{16} \sin 4 t, a_{12}(t)=a_{22}(t)=\frac{1}{16} \cos 4 t, \omega=\frac{\pi}{2}, \\
\alpha_{11}(t)=\alpha_{21}(t)=\frac{1}{8} \sin 2 t, \alpha_{12}(t)=\alpha_{22}(t)=\frac{1}{10} \sin 2 t, f_{1}(x)=f_{2}(x)=\arctan x, \\
\beta_{11}(t)=\beta_{21}(t)=\frac{1}{6} \sin 2 t, \beta_{12}(t)=\beta_{22}(t)=-\frac{1}{8} \sin 2 t, g_{1}(x)=g_{2}(x)=|x|, \\
I_{i}(t)=5 \sin 2 t, I_{2}(t)=3 \sin 2 t, \tau_{11}(t)=2, \tau_{12}(t)=3, \tau_{21}(t)=2, \tau_{22}(t)=4, \\
K_{11}(t)=K_{12}(t)=e^{-t}, K_{21}(t)=K_{22}(t)=e^{-2 t}, \mu_{1}=\mu_{2}=1, \nu_{1}=\nu_{2}=1,
\end{gathered}
$$

Take $\xi_{1}=\frac{1}{2}, \xi_{2}=\frac{1}{3}, \delta_{1}=\delta_{2}=0.8$. Clearly, $\int_{0}^{\infty}\left|K_{11}(u)\right| d u=\int_{0}^{\infty}\left|K_{12}(u)\right| d u=$ $1, \int_{0}^{\infty}\left|K_{21}(u)\right| d u=\int_{0}^{\infty}\left|K_{22}(u)\right| d u=\frac{1}{2}, \gamma=\frac{1}{2}$.

By simple computation, we have

$$
\begin{aligned}
& \sup _{t \in R}\left\{-c_{1}^{*}(t)+G_{i}\left[\frac { 1 } { \xi _ { 1 } } \sum _ { j = 1 } ^ { 2 } \left(\left|a_{1 j}(t)\right| \mu_{j}\right.\right.\right. \\
& \left.\left.\left.\quad+\left(\left|\alpha_{1 j}(t)\right|+\left|\beta_{1 j}(t)\right|\right) \nu_{j} \int_{0}^{\infty}\left|K_{1 j}(s)\right| d s\right) \xi_{j}\right]\right\} \\
& =-1.3054<-0.8<0
\end{aligned}
$$




$$
\begin{aligned}
& \sup _{t \in R}\left\{-c_{2}^{*}(t)+G_{2}\left[\frac { 1 } { \xi _ { 2 } } \sum _ { j = 1 } ^ { 2 } \left(\left|a_{2 j}(t)\right| \mu_{j}\right.\right.\right. \\
& \left.\left.\left.+\left(\left|\alpha_{2 j}(t)\right|+\left|\beta_{2 j}(t)\right|\right) \nu_{j} \int_{0}^{\infty}\left|K_{2 j}(s)\right| d s\right) \xi_{j}\right]\right\} \\
& =-2.5764<-0.8<0
\end{aligned}
$$

Hence, it follows that the assumptions (A1)-(A5) are satisfied. Therefore, according to Theorem 2 and Theorem 3, system (15) has one $\frac{\pi}{2}$-anti periodic solution which is globally exponentially stable.

\section{CONCLUSION}

In this paper, applying contraction mapping and fixed point theorem and differential inequality, we obtained some sufficient conditions for the existence, uniqueness and exponentially stability of anti periodic solution for fuzzy cellular neural networks with variable coefficients in leakage term. These conditions dependent on system parameters. The proposed results are less conservative than some recently known ones in the literature. The sufficient conditions obtained on existence and global stability of anti periodic solution are easily verifiable.

\section{REFERENCES}

[1] T. Yang, L. B. Yang, The global stability of fuzzy cellular neural networks. IEEE Trans. Circ. Syst. I, 43(1996), 880-883.

[2] T. Yang, L. B. Yang., C. W. Wu, L. O. Chua, Fuzzy cellular neural networks: theory. Proc IEEE Int Workshop Cellular Neural Networks Appl. (1996), 181186.

[3] K. Gopalsamy, Leakage delays in BAM. J Math Anal Appl, 325(2007), 1117-1132

[4] L. Peng, W. Wang, Anti-periodic solutions for shunting inhibitory cellular neural networks with time-varying delays in leakage terms, Neurocomputing, bf $111(2013), 27-33$.

[5] T. Huang, Exponential stability of fuzzy cellular neural networks with distributed delay. Phys. Lett. A, 351(2006), 48-52.

[6] Y. Q. Liu, W. S. Tang, Exponential stability of fuzzy cellular neural networks with costant and time-varying delays.Phys. Lett. A, 323(2004), 224-233.

[7] Y. Q. Liu, W. S. Tang, Existence and exponential stability of periodic solution for BAM neural networks with periodic coefficients and delays. Neurocomputing, bf 69 (2006), 2152-2160. 
[8] K. Yuan, J. Cao, J. Deng, Exponential stability and periodic solutions of fuzzy cellular neural networks with time-varying delays. Neurocomputing, bf 69(2006), 1619-1627.

[9] Q. Zhang, R. Xiang, Global asymptotic stability of fuzzy cellular neural networks with time-varying delays. Phys. Lett. A, 372 (2008), 3971-3978.

[10] Q. Zhang, W. Luo, Global exponential stability of fuzzy BAM neural networks with time-varying delays. Chaos, Sol. Fract., 42(2009), 2239-2245.

[11] Q. Zhang, L. Yang, D. Liao, Existence and exponential stability of periodic solution for fuzzy BAM neural networks with periodic coefficient, Ukrainian Mathematical Journal, 63, No 12 (2012), 1900-1915.

[12] C. Ou, Anti-periodic solution for high-order Hopfield neural networks, Comput. Math. Appl., 56 (2008), 1838-1844.

[13] Y. K. Li, L. Yang, Anti-periodic solutions for Cohen-Grossberg neural networks with bounded and unbounded delays, Commun. Nonlinear Sci. Numer. Simul., 14 (2009), 3134-3140.

[14] Q. Zhou, Anti-periodic solutions for cellular neural networks with oscillating coefficients in leakage terms, Int. J. Mach. Learn. $\&$ Cyber., 2016, DOI 10.1007/s13042-016-0531-1

[15] G. Q. Peng, L.H. Huang, Anti-periodic solutions for shunting inhibitory cellular neural networks with continuously distributed delays, Nonlinear Anal. RWA, 10 (2009), 2434-2440.

[16] J.Y. Shao, An anti-periodic solution for a class of recurrent neural networks, $J$. Comput. Appl. Math., 228 (2009), 231-237.

[17] P. L. Shi, L.Z. Dong, Existence and exponential stability of anti-periodic solutions of Hopfield neural networks with impulses, Appl. Math. Comput., 216 (2010), 623-630.

[18] L. Pan, J. Cao, Anti-periodic solution for delayed cellular neural networks with impulsive effects, Nonlinear Analysis: Real World Applications, 12 (2011), 30143027 .

[19] W. Wang, Anti-periodic solution for impulsive high-order Hopfield neural networks with time-varying delays in the leakage terms. Advances in Difference Equations,bf 2013 2013:273.

[20] Q. Zhang, L. Yang, J. Liu, Existence and stability of anti-periodic solutions for impulsive fuzzy Cohen-Grossberge neural networks on time Scales, Math. Slovaca, 64 (2014), 119-138. 
[21] Y. Li, J. Shu, Anti-periodic solutions to impulsive shunting inhibitory cellular neural networks with distributed delays on time scales, Commun Nonlinear Sci Numer Simulat.,16 (2011), 3326-3336.

[22] Z. Long, New results on anti-periodic solutions for SICNNs with oscillating coef?cients in leakage terms. Neurocomputing, 171(1) (2016), 503C509.

[23] H. Bao, On anti-periodic solutions for fuzzy BAM neural networks with constant delays on time scales, Dynamic Systems and Applications, 27, No. 3 (2018), 545-564.

[24] A. Jiang, Exponential Convergence for HCNNs with Oscillating Coefficients in Leakage Terms. Neural Process Lett., 43 (2016), 285-294.

[25] L. Berezansky, E. Braverman, On exponential stability of a linear delay differential equation with an oscillating coefficient. Appl Math Lett, bf 22(2009), 1833-1837. 